\title{
Morphed graphene nanostructures: experimental evidence for
}

\section{existence}

H. A. Calderon ${ }^{1}$, I. Estrada-Guel, ${ }^{2,3}$, F. Alvarez- Ramírez ${ }^{4}$, V. G. Hadjiev ${ }^{5}$, F.C. RoblesHernandez $z^{2 *}$

${ }^{1}$ Departamento de Física, ESFM-IPN, Ed. 9 Instituto Politécnico Nacional UPALM, Ciudad de Mexico, Mexico 07738

${ }^{2}$ Department of Mechanical Engineering Technology, University of Houston, Houston, TX 77204-4020, USA.

${ }^{3}$ Centro de Investigación en Materiales Avanzados, CIMAV, Miguel de Cervantes 120, Chihuahua, Chih. 31109, México.

${ }^{4}$ Instituto Mexicano del Petróleo, Eje Central Lázaro Cárdenas Norte 152, México D.F., México 07730

${ }^{5}$ Texas Center for Superconductivity and Department of Mechanical Engineering, University of Houston, Houston, TX 77204, USA

\footnotetext{
"To whom correspondence should be addressed. E-mail: $\underline{\text { fcrobles@uh.edu }}$
} 


\begin{abstract}
We present experimental evidence for morphed graphene nanostructures in which corrugated graphene layers are cross-linked covalently thus making either $\mathrm{sp}^{2}$ or $\mathrm{sp}^{3}$ type carbon allotropes. The structures are akin to recently derived by ab initio simulations [1] hexagonal carbon allotropes with R-3m crystal symmetry. The morphing effect comes from the hexagonal carbon rings bridging graphene-like layers. The key technological approach is by means of high-energy milling of amorphous carbon soot derived from fullerene production. The morphed graphene nanostructures are documented by a thorough characterization using HRTEM, XRD, and spectroscopies.
\end{abstract}

\title{
1. Introduction
}

Carbon nanostructures (CN's) are carbon allotropes with nanoscale dimensions, which include carbon nanotubes, fullerenes, polyaromatic molecules and graphene, nano onions, horns, torus, buds, cups and peapods [1,2]. Graphene is still the most interesting as compared to other CN's due to its potential for a wide variety of applications that are derived by its physical properties (thermal, optical, electrical, mechanical) [3, 4]. Another structure that is also well known is diamond. All the above mentioned structures have a $\mathrm{sp}^{2}$ bonding; except for diamond that is $\mathrm{sp}^{3}$ as well as one of the structures that we propose herein. In a way graphene is the foundation for all the graphitic $\left(\mathrm{sp}^{2}\right)$ structures [5]. The following methods are the most widely used to synthesized graphitic carbon: CVD with or without catalysts [6, 7], scotch tape or mechanical exfoliation (graphene) [4, 8], epitaxial growth [9] or chemical reduction [10]. On the other hand the synthesis of diamond is mainly thermomechanical (high temperatures, high pressures) [11]. 
The technological approach proposed in this work is purely mechanical and at near room temperature.

Identification of a new carbon allotrope is thus quite complicated due to the wide variety of known carbon phases and the pronounce effects of even subtle morphology changes as in the twisted layer graphene $[12,13]$. Nevertheless this paper describes different forms of evidence that lead to conclude that the product of the synthesis procedure in use (mechanical milling) indeed delivers a new allotrope. The raw material is an amorphous, yet specific, carbon soot which develops into a variety of phases during milling ranging from graphene (single, doublelayered) to diamond. More details will be given in forthcoming publications. Similar phases have been previously proposed from theoretical considerations and numerical calculations, but they have never been observed or synthesized [14]. In this work, is presented an in-depth characterization as evidence of this newly found allotrope.

\section{Experimental}

\subsection{Sample preparation}

The raw material is a commercial product called fullerene soot and it is a powdered carbon containing less than $1 \mathrm{wt} \%$ fullerenes (mainly $\mathrm{C}_{60}$ and $\mathrm{C}_{70}$ ). This soot is a byproduct of bulk fullerenes synthesized using the Krätschmer method [15] and a consequent leftover material once fullerene is extracted. In this work, batches of 2 grams have been milled using a SPEX 8000M mixer/mill. The milling times have been varied from 0 to $50 \mathrm{~h}$. The effect of milling media was evaluated using tungsten carbide, zirconia, alumina, and hardened steel vials. Prior to the characterization the materials prepared with steel milling medium were cleaned using a super magnet and an acid wash $\left(1 \mathrm{~h}\right.$ in $\left.6 \mathrm{M} \mathrm{HNO}_{3}\right)$ in an ultrasonic bath followed by an ethanol wash. 
The acid treatment and wash were repeated 5 times. This procedure is carried to prevent the presence of contamination (e.g. Fe) in the investigated materials.

\subsection{Methods}

The X-ray diffraction (XRD) analysis was carried out using a Siemens D5000 diffractometer under the following conditions: scan range $10-95^{\circ}, 0.0125^{\circ}$ step and $1 \mathrm{~s} / \mathrm{step}$. The Raman spectra were collected with a Horiba XploRA spectrometer using $638 \mathrm{~nm}$ and $532 \mathrm{~nm}$ laser excitations. Electron microscopy was performed in the TEAM 0.5 microscope of the NCEM-LBNL at $80 \mathrm{KV}$ and in low dose conditions. The dose rate in use $\left(15 \mathrm{e}^{-} / \AA^{2} \mathrm{~s}\right)$ allows taking 40 images at different defoci and performing an exit wave reconstruction procedure to obtain phase and amplitude images with atomic resolution. A phase image contains all information regarding interplanar spacing and nature of the atomic species.

\section{Results and Discussion}

Figure 1a shows the XRD spectra of soot and pristine $\mathrm{C}_{60}$ fullerite (molecular crystal). The soot is essentially amorphous except for some traces of fullerene; therefore, the reflections are not quite clear in the background [16]. The prominent reflection at approximately $25.4^{\circ}$ (in $2 \theta$ degrees) is the result of short-range ordered carbon structures. These structures correspond to a distribution of graphene flakes with small lateral size (almost clusters), loosely stacked at variable distances higher than that of graphite. Due to the absence of any other reflections typical of graphite, it is proposed that this soot has a limited arrangement in the " $c$ " direction. As per our previous results the average grain size in the " $c$ " direction is approximately $0.69 \mathrm{~nm}[16,17]$. Here we need to mention that this value is not the d-spacing but rather the size of each crystallite, 
which implies that each crystal is slightly larger than a single graphite lattice. Therefore, we cannot consider this as a crystalline material; instead, this a short-range structured or quasiamorphous material. The reflections in the XRD spectrum for fullerene are characteristic of the FCC structure (fullerite). From here it can be concluded that our soot does not have significant presence of fullerene and it is virtually amorphous.

Raman spectroscopy supports the XRD results (Figure 1b). There is an intense D band in the soot; typically this corresponds to nanosized or amorphous carbon. The G band suggests a graphitic structure and the weak 2D band, indicates that the honeycomb structure is only short range. This combination of features is common in carbon structures with limited order.

The fullerene soot observations under SEM are presented in Figure 1c showing scattered carbon particles in semi-spherical shapes. This morphology is characteristic for the residues after the fullerene is extracted from the evaporated carbon using the Krätschmer synthesis [15]. The soot has the appearance of agglomerates of clusters of amorphous or short range order carbon structures as seen in detail in Figure 1d. The HRTEM proves the amorphous nature of the soot, previously identified by XRD and Raman.

Samples milled from 1 to $50 \mathrm{~h}$ and characterization results were carried out to illustrate structural changes that occurred in the material. The effects of milling media were also investigated. The main goal is to identify the effect of milling in the synthesis of this new allotrope. In our previous work [17-22] we identified a crystalline carbon structure that do not correspond to any known phase of carbon. The identification was closed to a diamond-like structure based on the reported structures known as $2 \mathrm{H}$ and $4 \mathrm{H}$ in [23] diamond polytypes derived from numerical simulations. Here we present a further analysis of better prepared samples demonstrating these new carbon nanostructures that are neither diamond nor graphitic 
structures. Instead, we propose a new structure of carbon that we identify as morphed stack of graphene or morphed graphene.
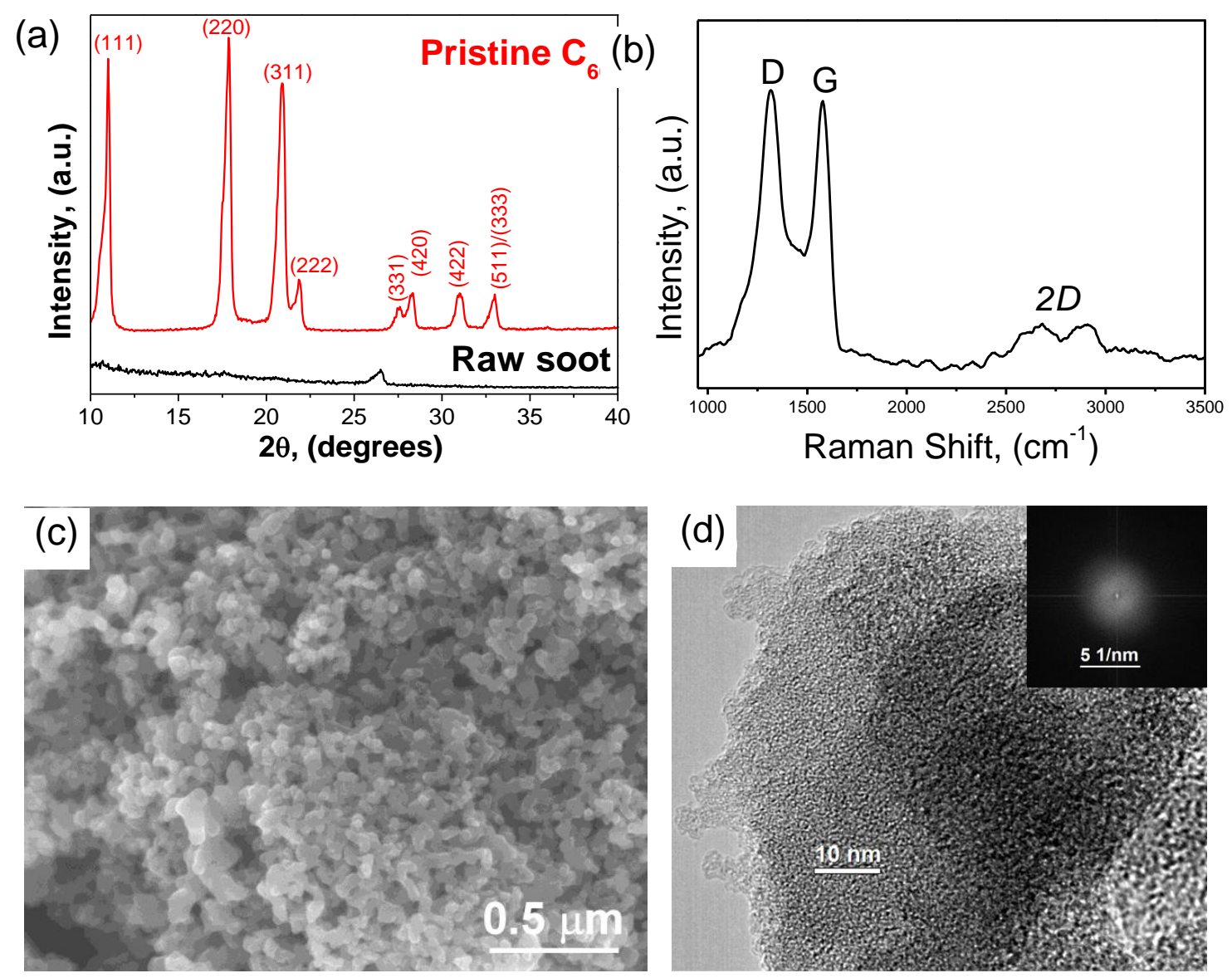

Figure 1. Characterization of the raw carbon soot by means of (a) XRD, (b) Raman Spectroscopy, (c) SEM and (d) HRTEM.

These carbon nanostructures were first identified in samples prepared using steel milling media and in order to confirm its existence, we used other milling media. The XRD results of the soot milled with various media are presented in SM 1. The XPS chemical analysis results are presented in Table 1. The use of all the milling media helps in the following ways: the morphed graphene is identified in all milling media; however, we selected steel media because it is the one that generates the least amount of contamination (see SM). The Fe contamination measured by 
XPS due to the steel media is less than 0.7 at $\%$ Fe for milling times of up to $20 \mathrm{~h}$. After $50 \mathrm{~h}$ of milling the contamination increased to approximately $3 \mathrm{wt} \%$. Figure 2 shows a HRTEM image of an as milled sample containing a variety of carbon microstructures. A larger area (collage) of the same sample is presented in SM 2.

Table 1. XPS results of the milled samples at various times. The presence of iron (Fe) is the result of contamination due to the milling media.

\begin{tabular}{|c|c|c|c|c|c|}
\hline \multirow{2}{*}{ Milling } & \multicolumn{3}{|c|}{ Elements (at\%) } & \multicolumn{2}{c|}{ Carbon Species (wt\%) } \\
\cline { 2 - 6 } time & C & O & Fe & $\mathrm{Sp}^{2}$ & $\mathrm{Sp}^{3}$ \\
\hline 0 & 94 & 6 & 0 & 95.8 & 4.2 \\
0.5 & 90.7 & 9.3 & 0 & 88.0 & 12.0 \\
2 & 88.4 & 11.6 & 0 & 87.1 & 12.9 \\
10 & 83.3 & 16.3 & 0.5 & 84.2 & 15.8 \\
20 & 83.9 & 15.5 & 0.6 & 81.8 & 18.2 \\
50 & 87.3 & 12.7 & 3.3 & 91.3 & 8.7 \\
\hline
\end{tabular}

The straight structures in Figure 2 are mainly graphitic phases and have the typical dspacing of graphite. The "curved or bent" region (Figure 2d) has deformed sections and the rest is comprised of amorphous carbon. The "bent" section displays a d-spacing that is different to that in graphitic structures. The morphed graphenes are first identified in samples prepared with milling times of less than $4 \mathrm{~h}$. The bending can be attributed to residual stresses resulting from 
permanent deformation during milling. This promotes the migration of carbon atoms in intermediate locations and consequent re-bonding. The re-bonding is reflected in the $\mathrm{sp}^{2} / \mathrm{sp}^{3}$ bond ratio that is measured in the XPS results (Table 1).

Figure $2 b, 2 c$ and $2 e$ represent graphitic structures with no apparent strain as confirmed by the d-spacing of 0.335 and $0.226 \mathrm{~nm}$ that are characteristic of the (002) and (101) planes in graphite [24] respectively. These values also correspond to the results of numerical simulations given in SM 3 and the respective Tables. Figure 2d shows different d-spacing in various sections and those differences are measured directly on the HRTEM image and further confirmed by measuring the reciprocal distances in the FFT-diffraction pattern. Figure 3a shows the FFT electron diffraction pattern from Figure $2 \mathrm{~b}$ that is comparable to that seeing in Figure $2 \mathrm{~b}$ and $2 \mathrm{e}$. In the three cases the d-spacing of the investigated particles matches perfectly with that of in graphene or graphitic carbon. The differences in d-spacing calculated using the FFT are less than $1 \%$ that is attributed to a systematic error. Therefore, here we demonstrate that we can produce graphitic carbon and double, triple or multi layered graphenes can be produced by mechanical milling.

A more detailed analysis of the differences in d-spacing for the identified reflections is presented in Figure 3-4. The FFT-diffraction patterns were generated in the locations identified in Figure $2 \mathrm{~b}$-e and their respective FFT are given in Figure $2 \mathrm{f}-\mathrm{g}$. The FFT given in Figure $2 \mathrm{~g}$ corresponds to locations Figure $2 \mathrm{~b}, \mathrm{c}$ and e that is used as example due to similarities among the three locations and with the aim to simplify this manuscript. On the other hand, Figure $2 \mathrm{~h}$ is the FFT for location Figure 2d. The FFT in Figure $2 \mathrm{~g}$ confirms the presence of typical graphitic carbon; however, Figure $2 \mathrm{~h}$ show the evidence of this new allotrope. The d-spacing of each 
reflection is given within the FFT with the identification of the respective plane based along with its corresponding miller indices and phase.

Figure 3 presents the typical atomic planes that should be expected in in graphitic carbon. On the other hand in Figure 4 the FFT-diffraction patterns have planes with a unique d-spacing, which does not correspond to any known graphitic structures. Some of the d-spacing in the identified structure is similar to that computationally derived in and proposed in [14] known as Rh6-II. Hitherto, to the best of our knowledge we have not identified in the literature the physical evidence of this allotrope as presented herein. The exact d-spacing for those structures was determined directly from observations on the HRTEM images and further confirmed by measurements carried directly on the FFT-diffraction patterns. Figure 3-4 are the respective IFFT images resulting from the diffracting planes observed in Figure $2 \mathrm{f}-\mathrm{g}$ with the respective IFFT for each of the characteristic planes for the indicated d-spacings. The inset in every image is the exact location to reconstruct the IFFT.

Figure 4 shows a detailed analysis of Figure $2 \mathrm{~d}$ and $2 \mathrm{~g}$ that is not typical of graphitic carbon. This is a plastically deformed area showing bent regions where this new allotrope forms during milling. The interatomic distances in the bent region vary from $3.43 \mathrm{~nm}$ to $3.73 \mathrm{~nm}$ apparently as a the result of plastic deformation. The reciprocal distance measured using the FFT pattern clearly show values that are comparable to those from the XRD simulations for the Rh6-II structure. The FFT values are more precise because they are the average of all the atoms and planes in the analyzed region. For further evidence we de-construct the FFT to separate each plane based on its characteristic d-spacing as per the simulated Rh6-II structure (consult the SM). This process is conducted to give a clear view of the atomic distributions for each plane. A larger area, "collage", of the particles presented in Figure 2-4 is given in SM 2 where is possible 
to observe a large density of the graphitic carbon and the bent regions. This image is presented to demonstrate that this is not a simple occurrence in or products.

Figure 4 shows a simulation analysis carried on the FFT diffraction pattern in Figure 2d. A number of different d-spacings, $0.168,0.173,0.216$, and $0.354 \mathrm{~nm}$, are found to match those calculated for the Rh6-II structure (see simulated XRD in SM 3 and 4). The respective diffraction planes for the abovementioned d-spacings are: (211), (220), (300), and (110). Those results match the structure known as Rh6-II. The simulated XRD patterns and crystallographic planes for Rh6 and Rh6-II are presented in the SM 3-4 along with their calculated d-spacing and atomic planes. The evidence for morphed graphene structures as Rh6-I and Rh6-II presented herein using HRTEM is further supported by XRD characterization.

We conclude from Figure 2 that the straight areas are more likely to be similar to graphitic carbon. While the bent areas are structures with dissimilar d-spacing and they are an intermediate state to synthesize this new carbon allotrope. In the following sections we present in more detail the other particles clearly showing a new crystal structure. The particles presented next are standalone in literally stress free regions and completely detached from the graphitic carbon. Further examples are given in the SM 5-6. The analysis presented next corresponds to specific particles that seem to match with the structures known as Rh6 and Rh6-II. 

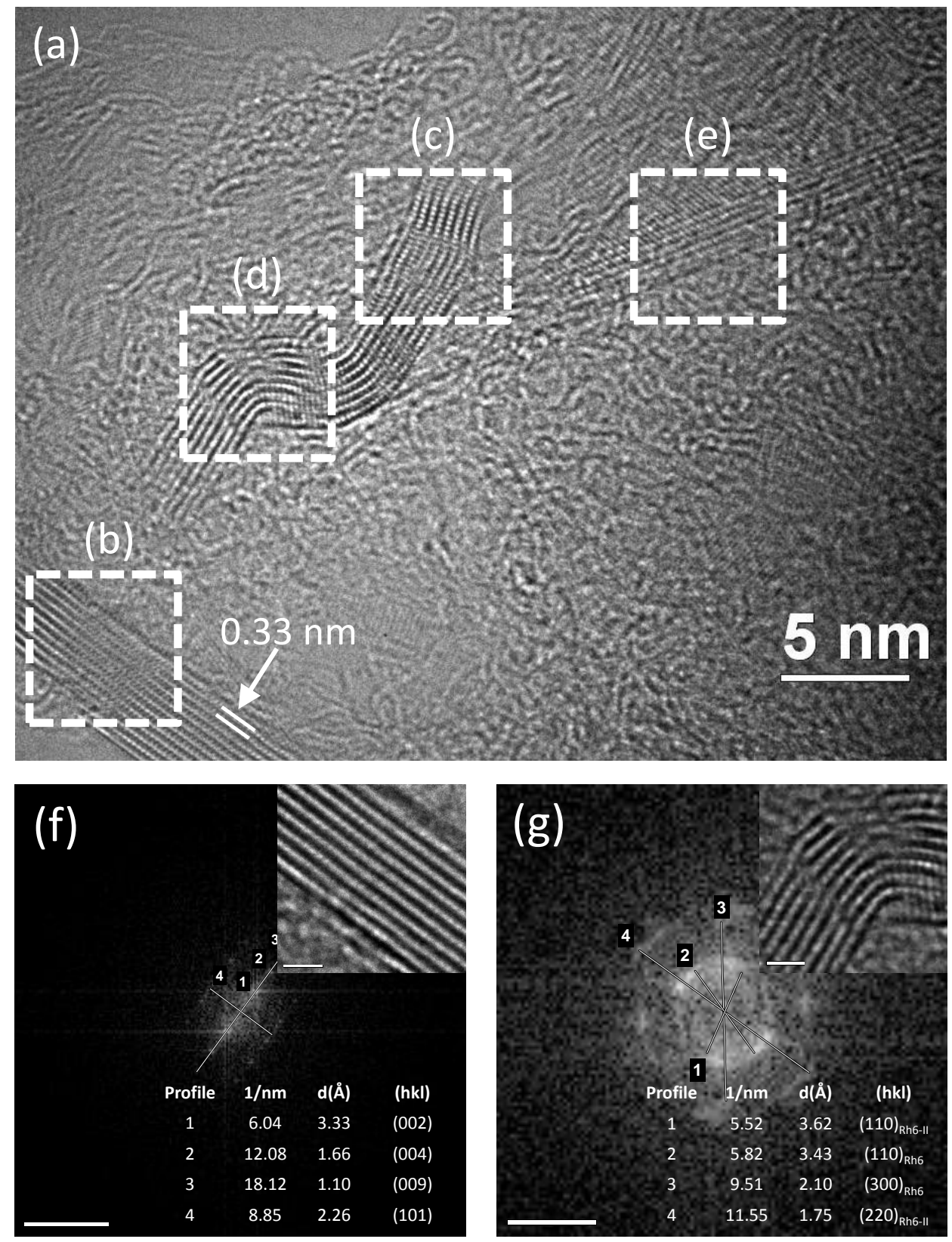

Figure 2. HRTEM micrograph of a carbon soot milled sample for $2 \mathrm{~h}$. In (a) is presented the morphology of relaxed and highly deformed structures of graphitic carbon. A characteristic FFT pattern for the regions identified as (b, $c$ and $e$ ) is given in ( $f$ ) and the inset correspond to the IFFT. The FFT for the region (d) is given in (g). The insets are the IFFT for the respective FFT patterns. 

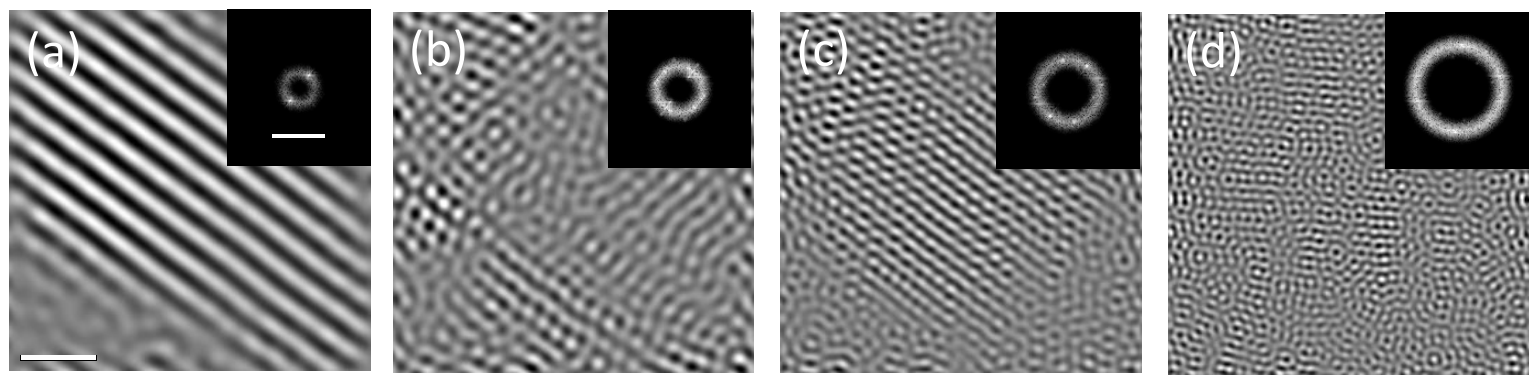

Figure 3. (a-d) FFT of Zone b in Figure 3. Figures (a-d) are filtered images of selected diffracting planes corresponding to the following are the respective graphitic planes for the respective IFFT images: (a) (002), (b) (004), (c) (009), and (d) (100). Note the planes presented here are identical to those from Figures $2 \mathrm{c}$ and $2 \mathrm{e}$.
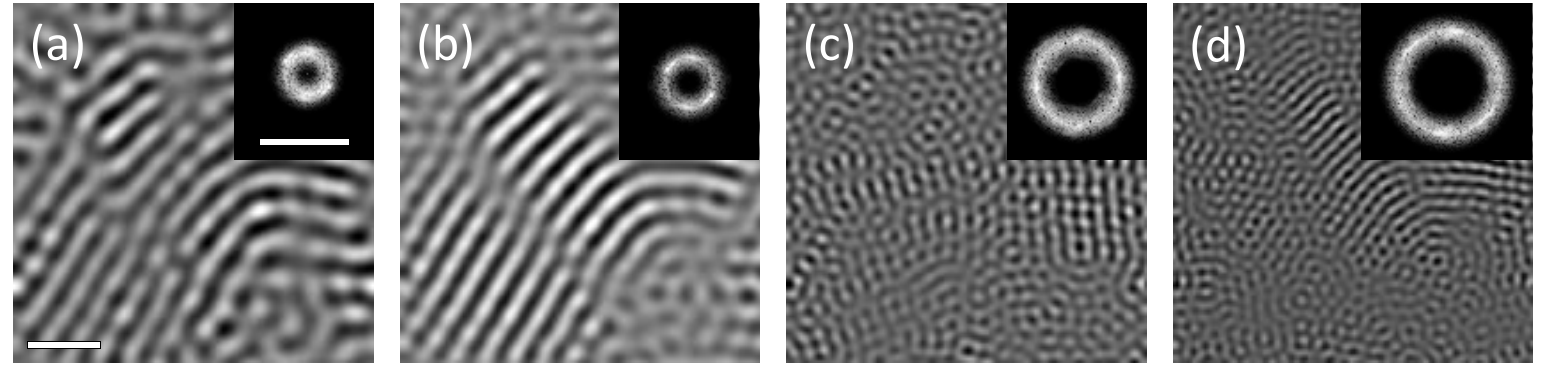

Figure 4. (a-d) FFT of Zone d in Figure 2. Figures (a-d) filtered images of selected spots showing their interplanar distances from the respective reflections as indicated in Figure $2(\mathrm{~g})$ for the RH6-II structure. The following are the respective planes for the RH6-II structure: (a) (211), (b) (220), (c) (300), and (d) (110).

Figure 5 shows results of $\mathrm{X}$ ray diffraction, in Figure $5 \mathrm{a}$, the $\mathrm{x}$ ray diffraction pattern is shown as a function of milling time. Figure $5 \mathrm{~b}$ shows the signature of new carbon structures in the XRD results. Given the small dimensions of the nanostructures embedded in a predominately amorphous mass in the material slice shown in Figure 2, one should not expect 
to record a complete set of XRD reflections. That is why the XRD results are intended only to confirm the dominant d-spacing observed with HRTEM but in this case at a macroscopic length scale. Figure 5 displays the evolution of the $25.5^{\circ}$ reflection with milling time as well as the development of a number of reflections between $36^{\circ}$ and $50^{\circ}$. The $26.3^{\circ}$ reflection broadens after $1 \mathrm{~h}$ of milling, but longer times seem to extinct this reflection and a new one develops at lower angles; implying a lattice enlargement. This phenomenon is in clear agreement with our simulations (SM 3) for the Rh6 and/or Rh6-II structures. The new reflection is observed first after $2 \mathrm{~h}$ of milling at $24.85^{\circ}$, and it stays at this location for up to $30 \mathrm{~h}$ of milling. This reflection corresponds to the (110) plane for both, the Rh6 and the Rh6-II. Due to the width of the band, it is not possible to discern if it correspond to the Rh6 or Rh6-II structures. Instead, we believe that our carbon has a blend of such structures. For this reason atomic resolution on HRTEM is mandatory for this work. Longer milling times ( $>30 \mathrm{~h})$ result in the decay in intensity of the (110) reflections for Rh6 and/or Rh6-II; possibly because either phases are mechanically damaged (e.g. deformed) or because carbon reacts with the Fe debris from the milling media.

The analysis of XRD results within $36^{\circ}-50^{\circ} 2 \theta$-range shows a family of new reflections corresponding to a mix of the reflections simulated for the Rh6 and Rh6-II structures [14]. The exact d-spacing for the simulated Rh6 and Rh6-II are given in SM 3-4. The set of XRD reflections shown in Figure $5 \mathrm{~b}$ confirms the presence of morphed graphene nanostructures with carbon bonding and arrangement of the Rh6 and Rh6-II crystals. Here is important to point out that the number of reflections present in the XRD patterns match closely to those in the simulated or the Rh6 and Rh6-II structures, including the new reflection(s) at approximately $24.8^{\circ}$ 

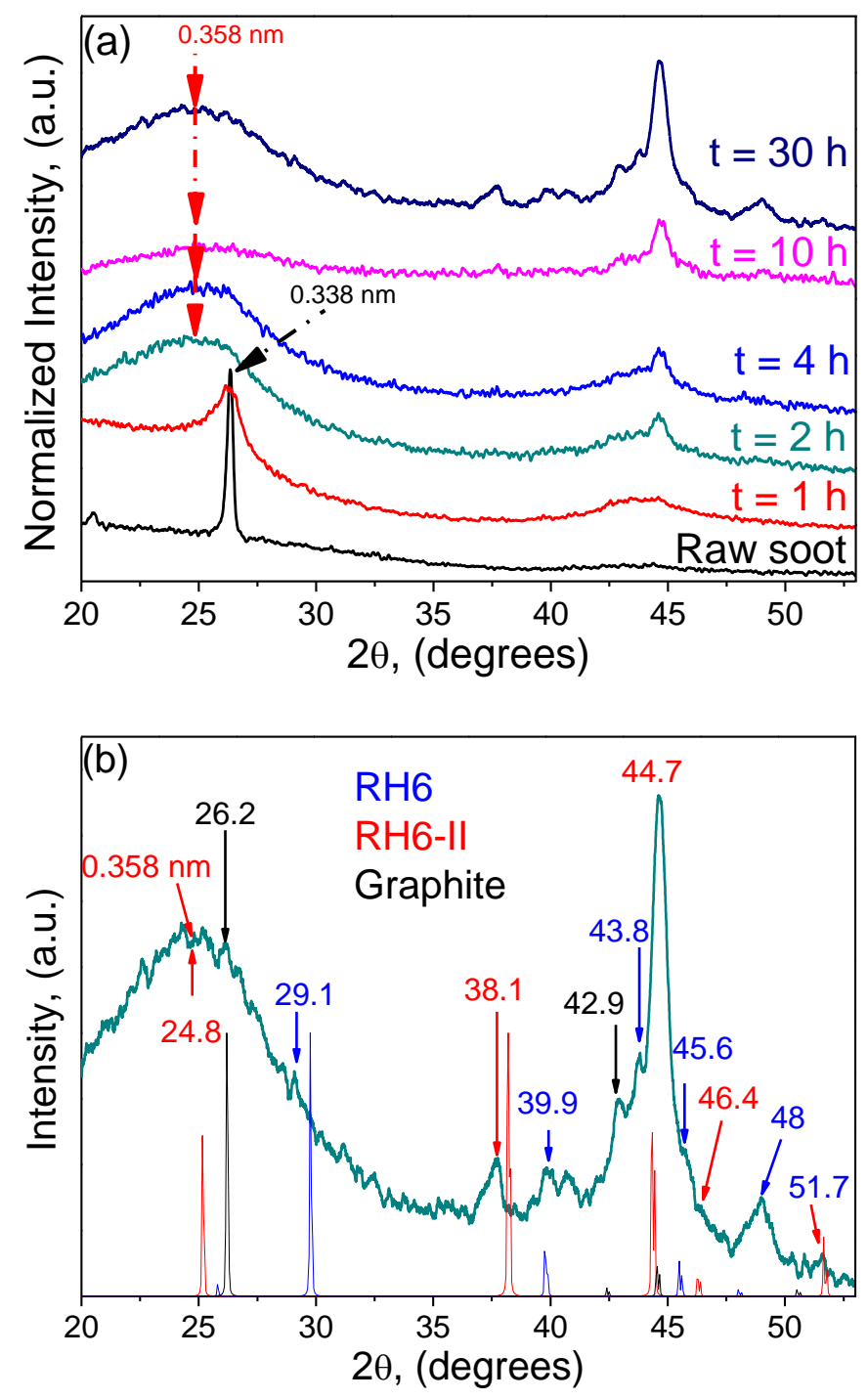

Figure 5. (a) Experimental XRD results for the milled soot from 1 to $30 \mathrm{~h}$, in (b) is presented the XRD of the samples milled for $30 \mathrm{~h}$ and index using the results of our numerical simulations for Rh6 and RH6-II (see SM). The sharper reflections in the background correspond to the simulated XRD spectra for graphite (black), Rh6 (blue) and Rh6-II (red) microstructures. Colors are available in the electronic version. 
The most intriguing observation is the existence of a morphed structure of Rh6-II type. While the morphing from graphene to Rh6 preserves the $\mathrm{sp}^{2}$ bonding, the one to Rh6-II rotates the existing bonds and makes new ones thus changing the bonding character from $\mathrm{sp}^{2}$ to $\mathrm{sp}^{3}$. The development of this transformation into the morphed graphene is clearly identified by XPS where we reach the maximum presence of $18.25 \mathrm{wt} \% \mathrm{sp}^{3}$ bonding at $20 \mathrm{~h}$ of milling. Based on the XPS results we have an approximate ratio between $\mathrm{sp}^{2}$ and $\mathrm{sp}^{3}$ of $4.5: 1$. Some of the $\mathrm{sp}^{2}$ belongs to the amorphous carbon and part of the $\mathrm{sp}^{3}$ is attributed to dangling bonds; perhaps $\sim 4.2$ $\mathrm{wt} \% \mathrm{sp}^{3}$ or slightly more that is measured in the raw sample. The increase in $\mathrm{sp}^{3}$ is an evidence of the changing nature of carbon during the morphing process. This proves that the milled carbon is not purely graphitic anymore. Yet, there is a critical time (e.g. 20h) or milling severity that allows to maximize the presence of $\mathrm{sp}^{3}$ and after that it decays. We attribute this decay to a potential amorphization of the allotrope or a reaction with Fe (contamination).

Further evidence of the combined presence of the Rh6 and Rh6-II nanostructures is given in Figure 6. In this case we are observing samples with larger milling time (more than 10h). The HRTEM image clearly reveals two regions where the main planes have a unique d-spacing of 0.344 and $0.356 \mathrm{~nm}$ (locations $i$ and $i i)$. In this analysis we measure the d-spacing directly from FFT diffraction patterns that as discussed previously it is more precise approach than direct measurements on HRTEM images. The FFT patters correspond to d-spacing along the areas identified as $(i)$ and (ii).

In location $(i)$ we identified two d-spacing one of 0.344 and the second of $0.362 \mathrm{~nm}$. Those two distances are attributed to a convoluted mix of the Rh6 and the Rh6-II structures respectively. Calculated FFTs are given in Figure 6 the respective IFFT for selective diffracting planes for each FFT are provided in Figure 7. . The Miller indexing in the FFT (Figure 6) 
corresponds to those obtained in the simulated XRD The d-spacing among the simulated XRD and the experimental evidence allows a clear identification showing a unique correlation among the results. The d-spacing of $0.356 \mathrm{~nm}$ is unique to the Rh6-II structure; however, it is similar to the lattice parameter for diamond; yet, the $0.232,0.202$, and $0.193 \mathrm{~nm}$ not typical of diamond and are characteristic of the Rh6-II phase.

Further evidence of the existence of the morphen graphenes (Rh6 and Rh6-II) is given in SM 4-5 where the experimental HRTEM images show a perfect match against those simulated using $a b$ initio. The experimental images in SM6 correspond to those given in Figures 5a and 5b. The planes presented herein are identify by HRTEM and confirm by XRD, and ab initio simulations for the Rh6 and Rh6-II structures. The XRD results (Figure 5) are also in good agreement with the expected for Rh6 and Rh6-II structures XRD patterns. Particularly interesting is the reflection at $24.8-2 \theta^{\circ}$ (Figure $5-\mathrm{XRD}$ ) that extinguishes at short milling times and a new reflection at $26.2-2 \theta^{\circ}$ becomes dominant. This new reflection is typical of the morphed graphenes. 

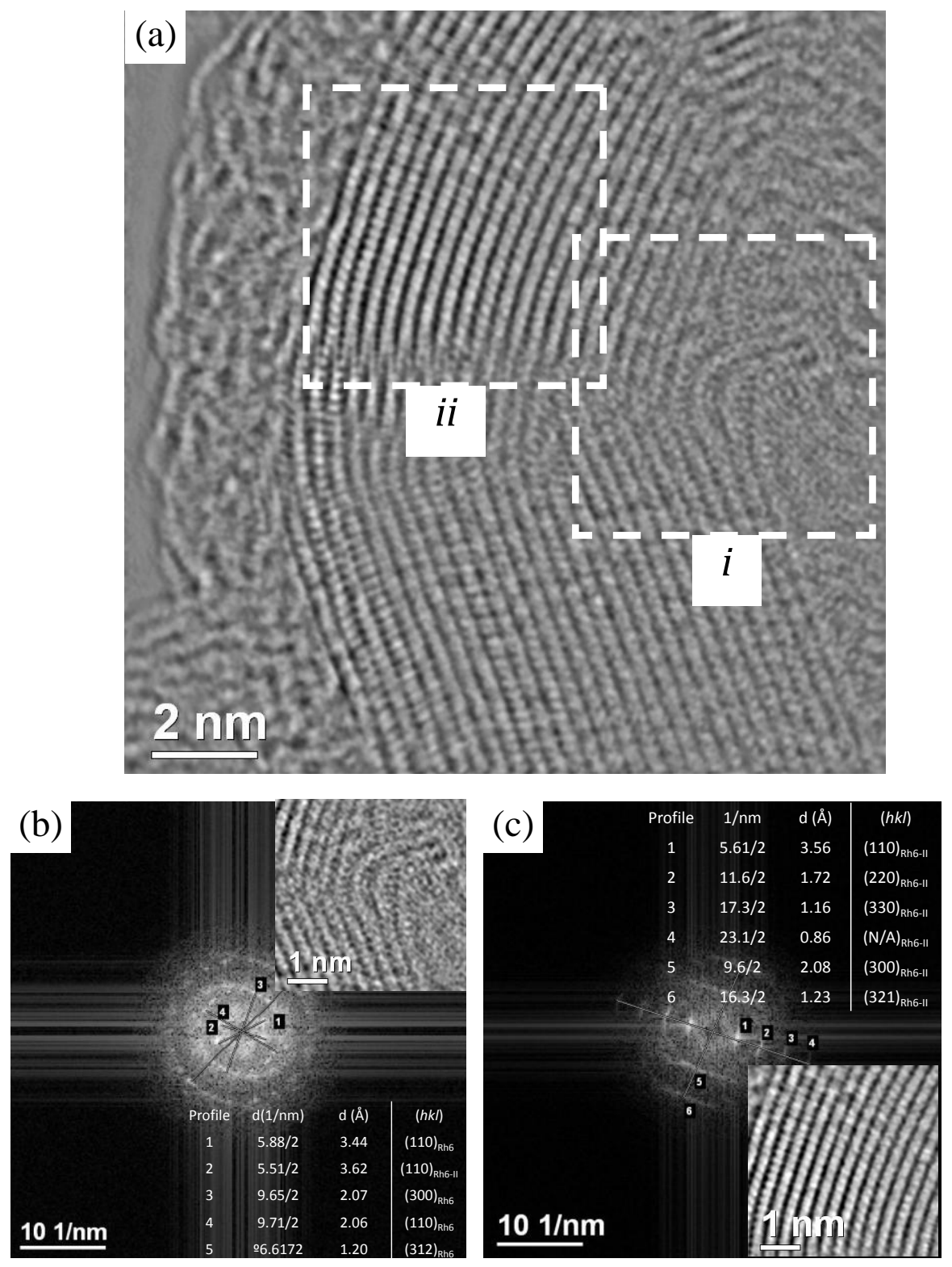

Figure 6. (a) HRTEM area with a combined presence of the Rh6 and Rh6-II particles. The doted squared in (a ) as (i) and (ii) were used to simulate the FFT-diffraction patterns in (b) and (c) respectively. The respective insets in each image correspond to the IFFT of the respective diffraction patterns. The numbers in the FFT patterns and the tables correspond to the identification of the respective planes for each structure. 
In the case of the Rh6 and Rh6-II structures the main difference is the plane (110) that is 0.345 and $0.351 \mathrm{~nm}$ respectively. As discussed before, this distance cannot be identified by means of XRD because of the width of the reflections (Figure 5); nevertheless, our evidence shows that this is not graphite. We are not the only group that reports the evidence of this dspacing. Further experimental evidence is included in SM 5-6 along with the respective ab initio simulated HRTEM images. Other groups, have observed similar structures in mechanically deformed graphenes [25] and so far has been attributed to chemical re-arrangements of carbon with hydrogen and oxygen. 

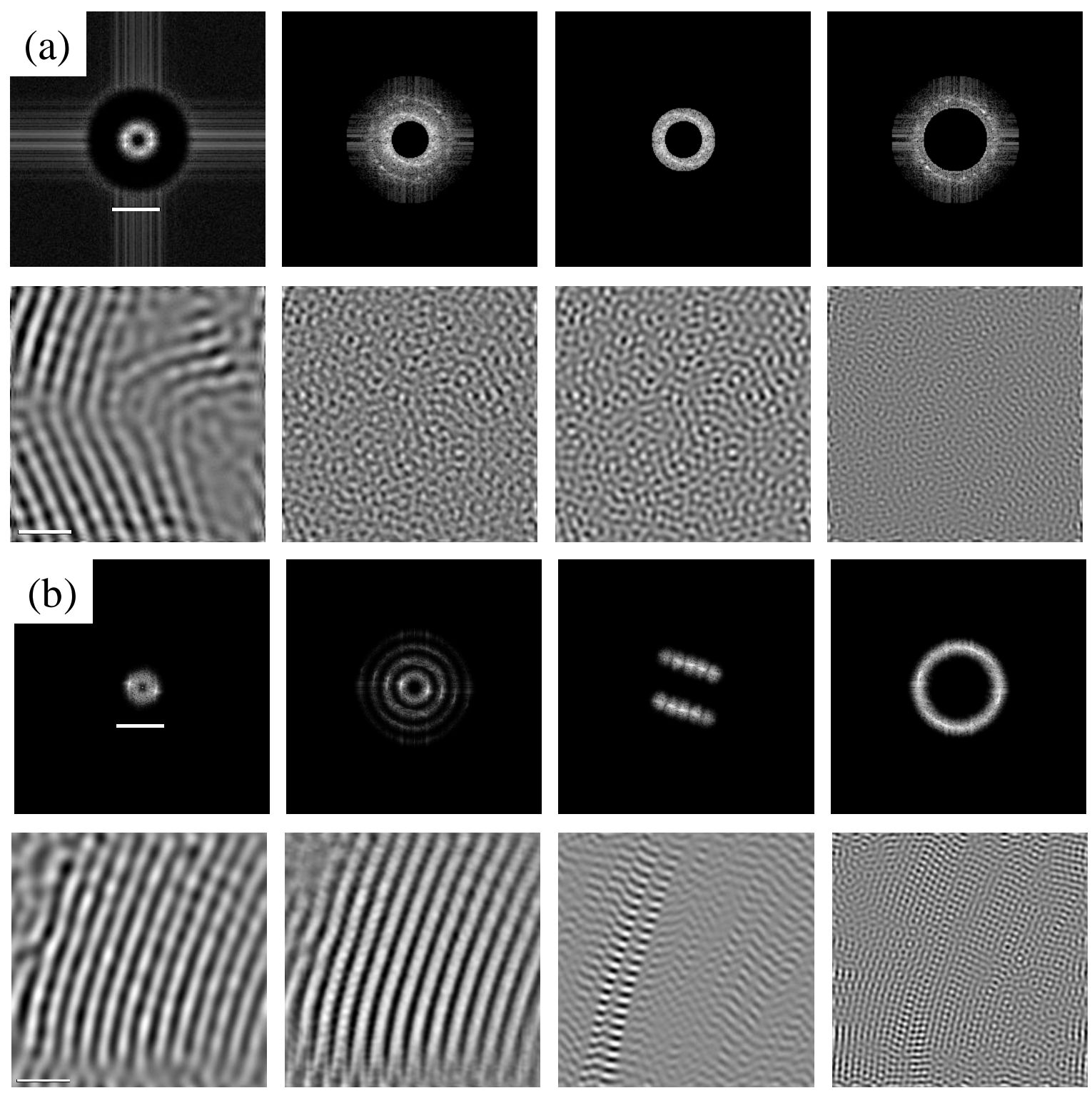

Figure 7. FFT and IFFT for the (a) Rh6 and (b) Rh6-II particles identified in Figure 6. The insets correspond to the simulated images for the respective allotropes.

The evolution of the morphed graphenes synthesized from milled soot can be summarized as follows: amorphous soot, graphene (2-3 layers), graphitic carbon (4-10+ layers) and then morphed graphene. The graphitic layers and the graphene structures are the result of welding during milling [26]. The morphed graphenes initiate their synthesis at relatively short milling 
times in highly stressed regions ("bents") and for larger milling times we found these particles "standalone" fashion. The presence of this new allotrope is mainly found in samples milled between 4 and $20 \mathrm{~h}$. The morphed graphenes synthesis and evolution is sketched as proposed in Figure 8. Shorter milling times usually result in the synthesis of graphene and graphitic carbon, and more than $20 \mathrm{~h}$ of milling further transform the morphed graphenes into diamond that is discussed in our future work.

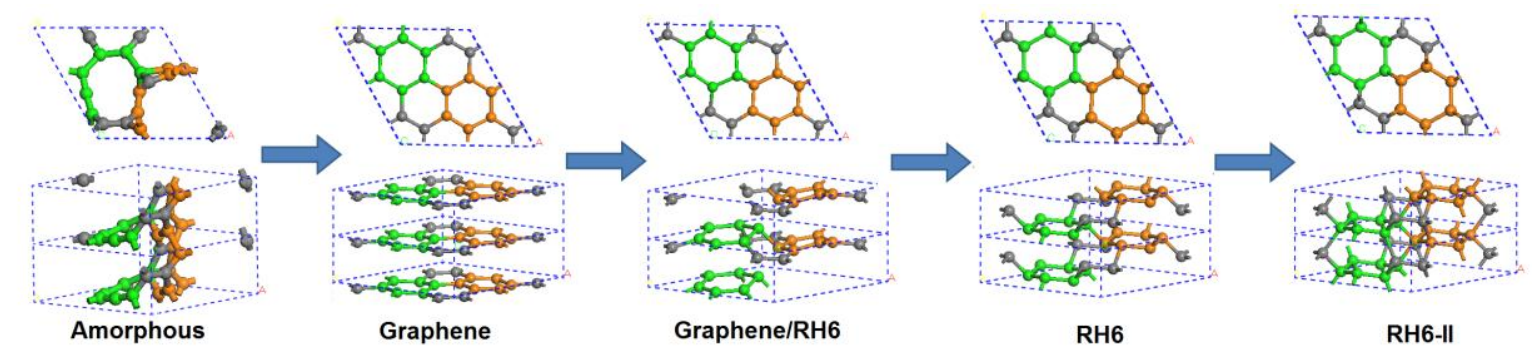

Figure 8. Mechanical synthesis of morphed graphenes.

On a final note we discuss the feasibility for using optical spectroscopic methods for characterization of the morphed graphene nanostructure. For example, Raman spectroscopy has proven to be one of the most successful techniques for characterization of carbon allotropes. On the other hand, since all carbon allotropes are strong Raman scatters, those that are present in a carbon sample in amount below certain threshold sometimes cannot be resolved. Part of the problem comes also from the overlapping Raman bands of different carbon allotropes. The carbon materials with different degree of amorphization produce not only broad Raman bands around the characteristic D and G peaks of graphite but also a relatively high scattering background. This background and broad $\mathrm{D}$ and $\mathrm{G}$ bands sometimes pose a detriment for resolving weak Raman peaks. That is the case of the materials presented in this work, which 
contain relatively small amount of morphed graphene nanostructures embedded in amorphous carbon. Nevertheless, we present in the SM 6 the results of a DFT simulation of the Raman active modes and intensity of Rh6 and Rh6-II structures and are compared to those of graphite and diamond to show that those phases can be clearly identified once a more abundant amount of the nanostructures material is produced. And the respective Raman simulated spectra is given in SM 6.

The DFT calculations were performed on the ab initio plane-wave pseudopotential code CASTEP [27]. The GGA was used to account for exchange and correlation in the PerdewBurke-Ernzerhof (PBE) form [28]. Both atom positions and the unit cell were relaxed to minimize the atomic forces and the total energy, such that forces were converged to $0.001 \mathrm{ev} / \mathrm{A}$ and stress residual to $0.002 \mathrm{GPa}$. A plane-wave cutoff of $310 \mathrm{eV}$ was used, and Brillouin-Zone integrals were performed using a 4x4x10 Monkhort-Pack grid [29]. The Raman spectra for all the carbon polymorphs were obtained from CASTEP package using Norm-conserving pseudopotentials (energy cut-off $310 \mathrm{eV}$ ) and the PBE-GGA exchange correlation functional.

\section{Conclusions}

We have presented experimental evidence for morphed graphene nanostructures in which the carbon-carbon bonding is predominantly $\mathrm{sp}^{2}(\mathrm{Rh} 6)$ or $\mathrm{sp}^{3}$ (Rh6-II) type. These nanostructures were synthesized form carbon soot with specific morphology using high-energy mechanical milling methods at room temperature. We believe that a future increase in production yield of these morphed graphene nanostructures is feasible and it would pave the way for new applications in structural composites. 
Acknowledgments IEG thanks the CONACYT support under project 169262. VGH work was supported by the State of Texas through the Texas Center for Superconductivity (TcSUH) at the University of Houston. HACB whishes to acknowledge the use of electron microscopes at NCEM of the Molecular Foundry (Lawrence Brekeley National Laboratory), which is supported by the Office of Science, the Office of Basic Energy Sciences, the U.S. Department of Energy under Con- tract No. DE-AC02-05CH11231. HACB also acknowledges CONACYT (grant 129207 and 148304) and IPN (COFAA).

\section{References}

1. Umadevi, D. and G.N. Sastry, Molecular and Ionic Interaction with Graphene Nanoflakes: A Computational Investigation of CO2, $\mathrm{H2O}, \mathrm{Li}, \mathrm{Mg}, \mathrm{Li+}$, and $\mathrm{Mg} 2+$ Interaction with Polycyclic Aromatic Hydrocarbons. The Journal of Physical Chemistry C, 2011. 115(19): p. 9656-9667.

2. Shenderova, O., D. Brenner, and R.S. Ruoff, Would Diamond Nanorods Be Stronger than Fullerene Nanotubes? Nano Letters, 2003. 3(6): p. 805-809.

3. Warner, J.H., et al., Graphene fundamentals to applications. First edition. ed. 2013, Amsterdam: Elsevier. pages cm.

4. Choi, W. and J.-w. Lee, Graphene : synthesis and applications. Nanomaterials and their applications. 2012, Boca Raton: CRC Press. xv, 347 p., 8 p. of plates.

5. Guldi, D.M. and N. Martin, Carbon nanotubes and related structures : synthesis, characterization, functionalization, and applications. 2010, Weinheim: Wiley-VCH. xxii, 539 p. 
6. Eizenberg, M. and J.M. Blakely, Carbon Interaction with Nickel Surfaces - Monolayer Formation and Structural Stability. Journal of Chemical Physics, 1979. 71(8): p. 3467-3477.

7. Li, X.S., et al., Large-Area Synthesis of High-Quality and Uniform Graphene Films on Copper Foils. Science, 2009. 324(5932): p. 1312-1314.

8. Geim, A.K. and K.S. Novoselov, The rise of graphene. Nature Materials, 2007. 6(3): p. 183-191.

9. Berger, C., et al., Ultrathin epitaxial graphite: 2D electron gas properties and a route toward graphene-based nanoelectronics. Journal of Physical Chemistry B, 2004. 108(52): p. 19912-19916.

10. Stankovich, S., et al., Synthesis of graphene-based nanosheets via chemical reduction of exfoliated graphite oxide. Carbon, 2007. 45(7): p. 1558-1565.

11. Akaishi, M., H. Kanda, and S. Yamaoka, Synthesis of Diamond from Graphite Carbonate Systems under Very High-Temperature and Pressure. Journal of Crystal Growth, 1990. 104(2): p. 578-581.

12. Wang, Y., et al., Resonance Raman spectroscopy of G-line and folded phonons in twisted bilayer graphene with large rotation angles. Applied Physics Letters, 2013. 103(12): p. 123101.

13. Wang, Y., et al., Four-fold Raman enhancement of 2D band in twisted bilayer graphene: evidence for a doubly degenerate Dirac band and quantum interference. Nanotechnology, 2014. 25(33): p. 335201.

14. Wang, J.-T., et al., A New Carbon Allotrope with Six-Fold Helical Chains in all-sp2 Bonding Networks. Scientific Reports, 2014. 4: p. 4339. 
15. Kratschmer, W., et al., Solid C-60 - a New Form of Carbon. Nature, 1990. 347(6291): p. 354-358.

16. Fals, A.E., V.G. Hadjiev, and F.C.R. Hernandez, Multi-functional fullerene soot/alumina composites with improved toughness and electrical conductivity. Materials Science and Engineering a-Structural Materials Properties Microstructure and Processing, 2012. 558: p. 13-20.

17. Robles-Hernández, F. and H. Calderón. Nanostructured Al-Al4C3 Composites Using C_Graphite or C_Fullerene as initial reinforcements. in ICCE-16 Conference. 2008. Kungming, China.

18. Robles Hernández, F.C. and H.A. Calderón. Synthesis of fullerene by Spark Plasma Sintering and thermomechanical transformation of fullerene into diamond on $\mathrm{Fe}-\mathrm{C}$ composites. in MRS Conference 2010. Cancun, Mexico: MRS.

19. Fals, A.E., V.G. Hadjiev, and F.C. Robles Hernández, Multi-functional fullerene soot/alumina composites with improved toughness and electrical conductivity. Materials Science and Engineering: A, 2012. 558(0): p. 13-20.

20. Fals, A.E., V.G. Hadjiev, and F.C. Robles Hernández, Porous media reinforced with carbon soots. Materials Chemistry and Physics, 2013. 140(2-3): p. 651-658.

21. Fals, A.E., J. Quintero, and F.C.R. Hernández, Manufacturing of Hybrid Composites and Novel Methods to Synthesize Carbon Nanoparticles. MRS Online Proceedings Library, 2010. 1276: p. null-null.

22. Santana I.I., et al., Fullerene-Metal Composites: Phase Transformations During Milling and Sintering. Solid State Phenomena, 2011. 172-174: p. 727-732. 
23. Ownby, P.D., X. Yang, and J. Liu, Calculated X-Ray-Diffraction Data for Diamond Polytypes. Journal of the American Ceramic Society, 1992. 75(7): p. 1876-1883.

24. Garibay-Febles, V., et al., Production and characterization of (Al, $\mathrm{Fe}$ )-C (graphite or fullerene) composites prepared by mechanical alloying. Materials and Manufacturing Processes, 2000. 15(4): p. 547-567.

25. Bhowmick, S., A. Banerji, and A.T. Alpas, Role of humidity in reducing sliding friction of multilayered graphene. Carbon, 2015. 87: p. 374-384.

26. Gilman, P.S. and J.S. Benjamin, MECHANICAL ALLOYING. Annual Review of Materials Science, 1983. 13: p. 279-300.

27. Clark, S.J., et al., First principles methods using CASTEP. Zeitschrift für Kristallographie, 2005. 220(5/6/2005): p. 567-570.

28. Perdew, J.P., K. Burke, and M. Ernzerhof, Generalized Gradient Approximation Made Simple. Physical Review Letters, 1996. 77(18): p. 3865-3868.

29. Monkhorst, H.J. and J.D. Pack, Special points for Brillouin-zone integrations. Physical Review B, 1976. 13(12): p. 5188-5192. 